\section{Reproductive Hazards}

\section{REPRODUCTIVE HAZARD IN THE WORKPLACE AND ENVIRONMENT}

Pau-Chung Chen*. Department of Public Health and Institute of Occupational Medicine and Industrial Hygiene, National Taiwan University College of Public Health, Taipei, Taiwan

\subsection{6/oemed-2018-ICOHabstracts. 1231}

\section{Aim of special session}

1. Provide new knowledge to prevent reproductive hazards.

2. Build mutual networks among safety and health experts

${ }^{1}$ Soile Jungewelter, ${ }^{2}$ Imane EL Amri, ${ }^{3,4}$ Andrea HiddingaSchipper, ${ }^{5}$ Ayman Fahim, ${ }^{6}$ Teus Brand, ${ }^{7}$ Ching-Chun Lin

${ }^{1}$ Finnish Institute of Occupational Health, Helsinki, Finland

${ }^{2}$ Faculty of Medicine and Pharmacy of Casablanca, Casablanca, Morocco

${ }^{3}$ National Institute for Insurance against Accidents at workDepartment of Occupational and Environmental Medicine, Epidemiology and Hygiene, Monteporzio Catone (RM), Italy

${ }^{4}$ Arbo Unie, Utrecht, The Netherlands

${ }^{5}$ Faculty Of Medicine, Suez Canal University, Ismailia, Egypt

${ }^{6}$ Netherlands Centre for Occupational Diseases, Coronel Institute of Occupational Health, Academic Medical Centre, Amsterdam, The Netherlands

${ }^{7}$ Institute of Occupational Medicine and Industrial Hygiene, National Taiwan University College of Public Health, Taipei, Taiwan

\section{$1727 a$ IS OCCUPATIONAL NOISE EXPOSURE DURING PREGNANCY RELATED TO LANGUAGE ACQUISITION OF THE CHILD?}

\footnotetext{
1,2Soile Jungewelter* ${ }^{*}, 2$ Helena Taskinen, ${ }^{3}$ Eira Jansson-Verkasalo, ${ }^{1,4}$ Marija-Liisa Lindbohm, ${ }^{1}$ Jouko Remes, ${ }^{5}$ Minna Huotilainen, ${ }^{1}$ Markku Sallmén. ${ }^{1}$ Finnish Institute of Occupational Health, Helsinki, Finland; ${ }^{2}$ University of Helsinki, Helsinki, Finland; ${ }^{3}$ University of Turku, Department of Psychology and Speech, Turku, Finland; ${ }^{4}$ University of Tampere, Tampere, Finland; ${ }^{5}$ University of Helsinki, Cognitive Brain Research Unit, Helsinki, Finland
}

\subsection{6/oemed-2018-ICOHabstracts. 1232}

Introduction We examined the impact of noise exposure during pregnancy on the child's language acquisition at the age of one and two years.

Methods We conducted a cohort study among women working in the food industry, as kindergarten teachers, musicians, dental nurses and pharmacists with a new born child. At the age of one year the analyses included 408 mother-child pairs and at the age of two years 288. The mother filled the baseline questionnaire before the child was 12 months old, and the language acquisition questionnaire when the child was 12 months (Infant-Toddler Checklist, ITC) and 24 months (MacArthur Communicative Development Inventories, MCDI) old. An occupational hygienist assessed the noise exposure in three categories: no exposure, low exposure, moderate/high exposure. We analysed the data using linear regression.
Results At the age of one there were no statistically significant differences among the adjusted mean language acquisition ITC-scores of the children in different exposure categories. The adjusted scores among boys were 30.1 (95\% CI: 28.3 to 31.8 ) for no exposure, $29.7(27.4-32.0)$ for low noise, and 29.3 (26.7-31.9) for moderate/high exposure. Among girls these were 33.7 (31.9-35.5), 33.8 (31.3-36.4), and 33.6 (31.3-36.0), respectively. No associations were found in analyses of social communication, speech production and language comprehension. Noise exposure was associated with lower scores among kindergarten teachers. At the age of two mean MCDI-scores did not differ significantly between the noise exposure groups. The adjusted mean scores for expressive vocabulary among girls were 295 (95\% CI: 254 to 336) for no exposure, 303 (243-362) for low exposure, and 269 (212-326) for moderate/high exposure. Among boys the scores were 200 (154-246), 178 (111-246), and 225 (153-298), respectively.

Discussion We found no clear association between noise exposure during pregnancy and language acquisition among oneyear- or two-year-old children.

\section{7b PREMATURE BIRTH AND WORKING CONDITIONS: A PROSPECTIVE STUDY ABOUT 68 PREGNANT WOMEN}

${ }^{1}$ I El Amri ${ }^{*},{ }^{2} \mathrm{~F}$ Benbrahim, ${ }^{1} \mathrm{~B}$ Benali, ${ }^{1} \mathrm{~A}$ El Kholti. ${ }^{1}$ Faculty of Medicine and Pharmacy, Casablanca, Morocco; ${ }^{2}$ Faculty of Medicine and pharmacy, Rabat, Morocco

\subsection{6/oemed-2018-ICOHabstracts.1233}

Introduction The question about mediation between work and pregnancy is a real problem in industrialised countries, but in a country like ours, the issue is far away from being taken as a priority. Thus, we've chosen to conduct this prospective study about premature birth and working conditions trying to find a correlation between the two, while removing medical and obstetrical risk factors.

Methods The study was conducted in between January and December 2014. We've included women who had a job 6 months or more before the conception. To evaluate working conditions we've used the fatigue score developed by Mamelle. To evaluate the impact of working conditions on premature birth, we've monitored cervical length changes.

Results At first, we've gathered a selection of 68 pregnant women debuting their follow up between 11 and 13 weeks of amenorrhea plus 5 days. An acceleration of the modification of the uterine cervix was noted within the group of women having a «Mamelle» score of 3 .

Discussion The first epidemiological study bringing up the part of tiring working conditions in premature birth was conducted by Mamelle in France. After that, more authors, such as Mac Donald, got interested by the subject and implicated long working hours, rotating shifts and carrying heavy loads in the occurrence of premature birth. We took $18 \mathrm{~mm}$ as a limiting value for the length of the cervix under which intervention measures should be taken.

Conclusion Risk assessment of working conditions is the only deciding factor when it comes to the pursuit of work while being pregnant. The decision needs to be taken by both the attending and occupational physicians. 